\title{
Effects of neuromuscular training and strengthening of trunk and lower limbs muscles in women with Patellofemoral Pain: A protocol of randomized controlled clinical trial, blinded
}

Natália Camin Silva, Matheus de Castro Silva, Morisa Garcia Guimarães,

Manoela Beatriz de Oliveira Nascimento and Lilian Ramiro Felicio* (1)

\begin{abstract}
Background: Patellofemoral pain (PFP) often affects young women, and the etiology is multifactorial and poorly understood. Conservative intervention has been focused on risk factors or aggravating factors and is composed of hip- and knee-strengthening exercises, as this population often has muscle weakness and poor motor control during daily and sports activities. The objective of this study was to evaluate the additional effects of neuromuscular training in a conservative treatment of trunk-, hip-, and knee-muscle strengthening on pain, function, and kinematics of the trunk, pelvis, and lower limb in women with PFP.

Methods: This is a randomised clinical trial, controlled, blinded. Ninety women who are active and engage in physical activity up to twice a week will be recruited. All participants will undergo an individual physiotherapy assessment and then will be allocated randomly into two groups. Thereafter, both groups will undergo a 12-week intervention protocol: group 1 will perform strengthening exercises for the trunk, hip, and knee muscles, while group 2 will receive the same treatment, with the inclusion of neuromuscular training exercises on the fourth week. At the end of the intervention, the volunteers will be evaluated. The primary outcomes will be pain intensity (using a Visual Analog Scale: over the last month, squat $90^{\circ}$, and step of $26 \mathrm{~cm}$ during $1 \mathrm{~min}$ ), functional capacity (Anterior Knee Pain Scale and Activities of Daily Living Scale), and 2D kinematics of the trunk, pelvis, and lower limb during the single-leg squat. The secondary outcomes correspond to the isometric muscular strength of the lower limb and the level of satisfaction from the intervention.
\end{abstract}

Discussion: The present study was initiated on 28 January 2018 and is currently in progress, scheduled for completion in July 2019. The results of this study should contribute to the physiotherapeutic treatment of women with PFP by aggregating information on the benefits of adding neuromuscular training to strengthening of the trunk and lowerlimb muscles.

Trial registration: Registro Brasileiro de Ensaios Clínicos, ID: RBR-8c7267. Registered on 2 August 2017.

Keywords: Patellofemoral pain, Muscle strength, Neuromuscular training, Kinematics, Hip and knee

\footnotetext{
*Correspondence: lilianrf@ufu.br

Programa de Pós-Graduação em Fisioterapia na Universidade Federal de Uberlândia (UFU), Faculdade de Educação Física e Fisioterapia (FAEFI), Rua Benjamin Constant, 1286, Uberlândia, MG CEP: 38400-678, Brazil
}

(c) The Author(s). 2019 Open Access This article is distributed under the terms of the Creative Commons Attribution 4.0 International License (http://creativecommons.org/licenses/by/4.0/), which permits unrestricted use, distribution, and reproduction in any medium, provided you give appropriate credit to the original author(s) and the source, provide a link to the Creative Commons license, and indicate if changes were made. The Creative Commons Public Domain Dedication waiver (http://creativecommons.org/publicdomain/zero/1.0/) applies to the data made available in this article, unless otherwise stated. 


\section{Background}

Patellofemoral pain (PFP) is a common musculoskeletal impairment, with high incidence, involving adolescents, military personnel, and elite athletes [1], with an annual prevalence of $22.7 \%$ of the general adult population [1], being higher in women [1]. It is defined by pain in the anterior region of the knee, which is accentuated by performing activities that increase the load on the patellofemoral joint, such as squatting and going up and down the stairs [2, 3]. It is believed that PFP is a potentially contributing risk factor for the development of patellofemoral osteoarthritis [4-6].

The etiology of PFP is very complex, multifactorial $[7,8]$ and seems to involves the biopsychosocial aspects of each individual [9]. It is known that the population with PFP presents anxiety, depression, catastrophism, and fear of movement of the painful joint, and these are correlated with pain [10]. However, more studies are needed to better understand the role of psychological, social, cultural, and behavioral aspects in the development of PFP. In general, the biological factors are associated with abnormal joint load/stress [8].

According to a recent systematic review and meta-analysis of prospective studies [7], isometric/isokinetic weakness of the quadriceps is a strong risk factor for the development of PFP, and moderate evidence indicates that increased strength of the hip abductors is a predictor of PFP in adolescents. However, because of the complexity of PFP, prospective longitudinal studies are still needed to identify possible risk factors [7, 11].

It is known that the population with PFP has a deficit of strength and motor control of the trunk [8, 12-14], posterolateral musculature of the hip [8, 12-17], and quadriceps $[7,8,12,18]$. Some authors indicate a probable relationship between PFP and lack of appropriate control of lower-limb movement especially in strenuous activities [8, 14, 19-24], in which this motor-control deficit causes excessive dynamic valgus, increasing the lateralization of the patella and, thus, patellofemoral stress $[25,26]$. Thus, excessive dynamic valgus is a potentially predictive factor for PFP [26, 27]. In view of this, the use of a protocol that aims to improve the kinematics of the lower limb is indicated, thus minimizing patellofemoral stress [24].

Conservative treatment for PFP has been focused on trunk- and hip-muscle-strengthening protocols associated with strengthening of the knee musculature [2833 ], as pain reduction and motor function improvement were observed in these patients [28, 29, 34].

In view of the kinematic characteristics of this population, the integration of neuromuscular training to the strengthening protocol may be important. However, the evidence-base is poor and unclear [32, 33, 35-37]. We found only five studies that evaluated these phases of physical therapy in patients with PFP. One was a case report [32]; two studies observed the effect of motor control under dynamic alignment during gait [35, 36]; one study evaluated trunk and lower-limb kinematics following an isolated knee-strengthening and stretching program versus a hip-strengthening program associated with neuromuscular training [33]. Moreover, only one study evaluated the additional effect of neuromuscular training, concluding that the addition of neuromuscular training does not promote significant improvement in the kinematics of the trunk and lower limb [37]. However, the small sample size, relatively unchallenging motor-control protocol, and short, 4-week, intervention period could have influenced the results [37]. Therefore, ,studies that address neuromuscular training in the treatment of individuals with PFP are necessary.

Taking into account these aspects, the objective of this study was to evaluate the addition of neuromuscular training to the strengthening of the trunk, hip, and knee muscles on pain, functional capacity, and kinematics of the trunk, pelvis, and lower limb in patients with PFP. Our hypothesis is that, compared to the group that received only strength training, the group submitted to the protocol that combined neuromuscular strengthening and training would show greater improvement in pain, function, lower-limb and trunk kinematics, and strength of the hip and knee.

\section{Methods \\ Study design}

The present study is a blinded, randomized controlled, clinical trial with two parallel groups (Figs. 1 and 2). The trial was approved by the Human Rights Ethics Committee of the Universidade Federal de Uberlândia (UFU) under protocol number CAAE: 57621316.0.0000.5152. The study was registered at Registro Brasileiro de Ensaios Clínicos (ReBEC) (trial registration number, RBR-8c7267) and is being funded by the Coordenação de Aperfeiçoamento de Pessoal de Nível Superior (CAPES). It started recruiting patients on 28 January 2018, and data collection will likely be completed by July 2019. The evaluations will be performed before and after the 12-week intervention, and the variables observed are as follows: pain intensity, functional capacity, kinematics of the trunk, pelvis, lower limbs, and muscle strength.

\section{Participants and therapists}

The sample size calculation was performed using the mean difference and standard deviation error (based on outcomes of pain; function; kinematics of the trunk, hip, and lower limb during the single-leg squat; and strength of the hip and knee) of similar studies in the literature. The power of the test considered was $80 \%$ and the alpha was 0.05 , with a sample size of 45 per group. In this 


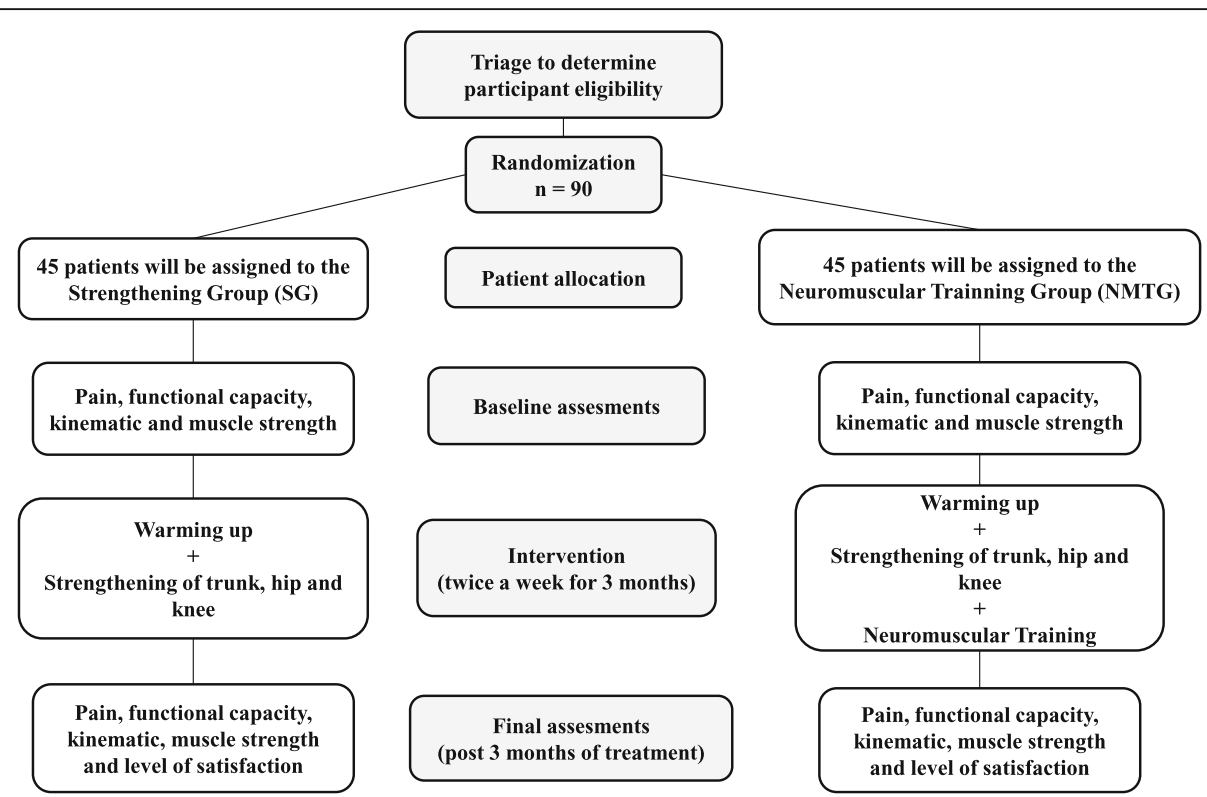

Fig. 1 Flowchart of the study design. (Figure adapted according to the model used by Dos Anjos Rabelo ND, et al. [37]. Authorization granted by the authorship and original publisher.)

analysis, the program BioEstat version 5.3 (Manaus, Brazil) was used.

A total of 90 active female individuals aged $18-30$ years who have anterior or retropatellar pain in the knee (with a Visual Analog Scale (VAS) score $\geq 3$ ) [2, 33] for at least the last 3 months (in two or more of the following activities: running, walking, jumping, climbing up and/or down stairs, remaining seated or kneeling for a long period of time, squatting, during isometric extension of the knee with $60^{\circ}$ of flexion, and during palpation of the medial or lateral facet of the patella) [33], and who perform any type of physical activity up to twice a week and/or are irregularly active $\mathrm{A}$ and $\mathrm{B}$ individuals according to the International Physical Activity Questionnaire (IPAQ), will be recruited [38]. The exclusion criteria are as follows: previous physical therapy treatment; history of knee surgery; patellar dislocation; ankle or hip injuries; meniscal or ligament injury; any other specific knee changes; tendinitis in the lower extremity; low back pain; sacroiliac joint pain; pregnancy; and the presence of any neurological, cardiovascular, and rheumatological changes or any changes that compromise the understanding of the procedure and affect physical therapy tests and treatment $[15,31]$.

These individuals will be recruited through posters set up on the campus of the Universidade Federal de Uberlândia (UFU), and by dissemination via social networks and radio in the city of Uberlândia, with the purpose of informing and arousing the participatory interest in the desired population.

This research will be developed in the Laboratório de Avaliação em Biomecânica e Neurociências (LABiN) and the Physical Therapy Clinic School of UFU, Brazil.

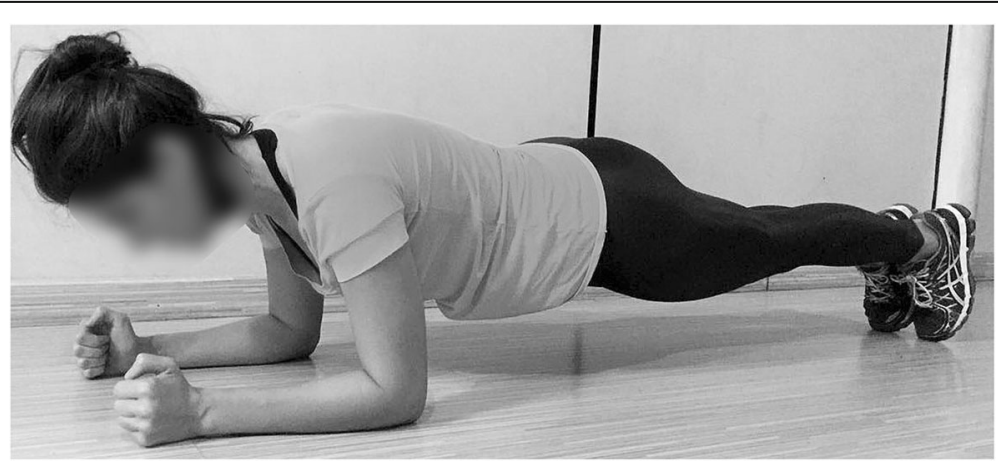

Fig. 2 Ventral Plank on a stable surface (1st-12th week). This exercise will be performed by the Strengthening Group (SG) and Neuromuscular Training Group (NMTG). (Originals photos and own authorship) 


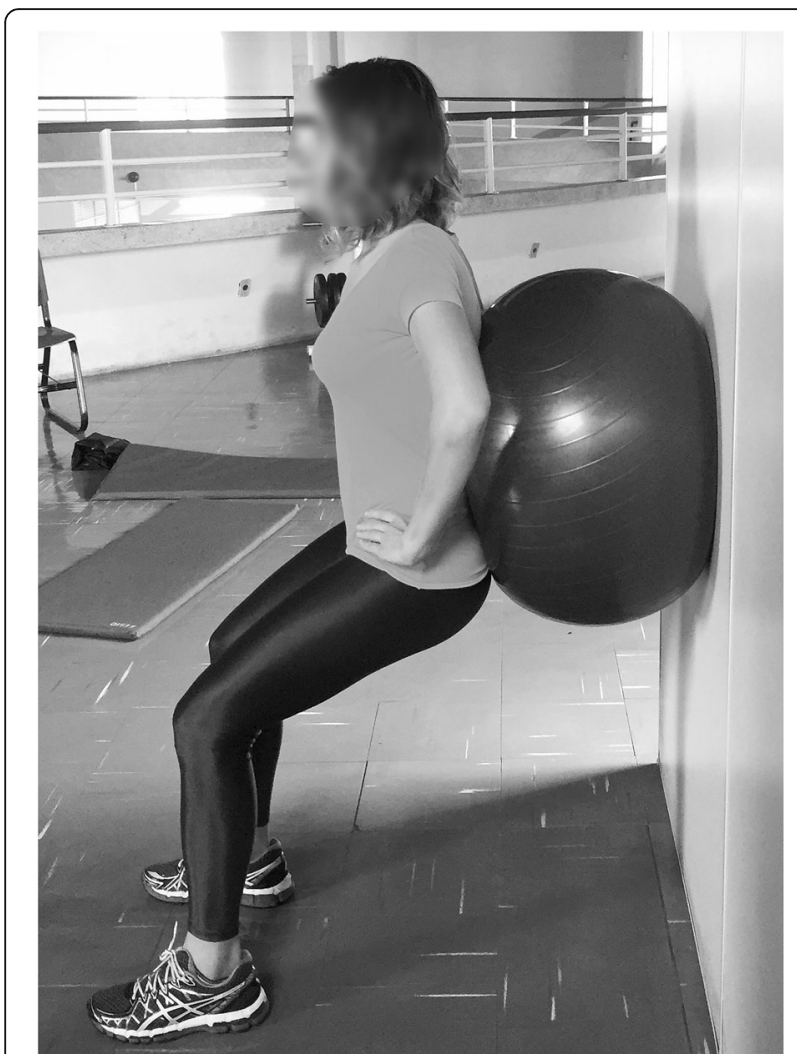

Fig. 3 Wall squat (1st-12th week). This exercise will be performed by the Strengthening Group (SG) and Neuromuscular Training Group (NMTG). (Originals photos and own authorship)

\section{Procedure}

All subjects will receive a written Consent Form to participate in this study. Thereafter, the participants will be submitted to an individual physiotherapeutic evaluation, and if they meet the inclusion criteria they will be randomly distributed into two intervention groups.

The randomization (fixed allocation of the simple type) will be conducted by an investigator who is not involved in the recruitment, evaluation, and/or treatment of participants. The randomization codes will originate from the RAND function of Microsoft Excel for Windows and will be inserted into sealed opaque envelopes and listed sequentially to hide the allocation. The envelopes will be opened by the same investigator who generated the codes.

The investigator responsible for the physiotherapeutic evaluation is blinded to the treatment allocation. Participants will be informed that they will receive physiotherapeutic treatment but will not know of the differences between the treatment groups, or the study hypothesis, and can be considered as blinded. Three physiotherapists were adequately trained to apply the intervention protocol. Each patient will be treated by only one therapist who is not involved in the physiotherapeutic evaluation and can be considered as blinded.

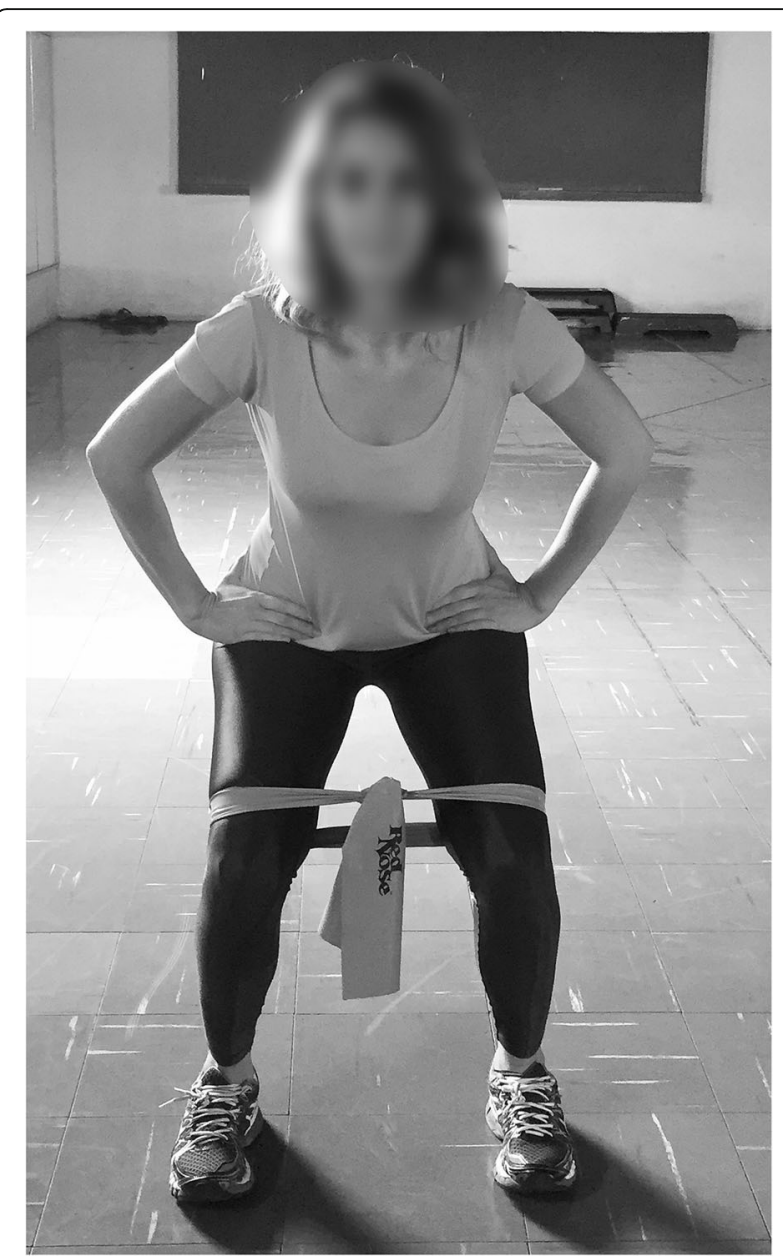

Fig. 4 Lateral Walk with elastic band (1st-12th week). 1st-2th week: elastic band strong average; 3st-5th week: elastic band strong; $6 s t-8$ th week: elastic band super strong; From the 9st week: elastic band extra strong. This exercise will be performed by the Strengthening Group (SG) and Neuromuscular Training Group (NMTG). (Originals photos and own authorship)

\section{Intervention/control}

Ninety patients will be randomly allocated into two groups: (1) Strengthening Group (SG): submitted to strengthening exercises of the muscle trunk, hip, and knee; and (2) Neuromuscular Training Group (NMTG): submitted to the same protocol as the SG, with the addition of neuromuscular exercises on stable and unstable ground.

Both groups will be submitted to physiotherapeutic treatment for a period of 12 weeks, performing two sessions per week, totaling 24 sessions, with a duration of 60-90 min per session. Each session will consist of 10 min of warming up on elliptical equipment, with a comfortable velocity for the patient and mild-moderate intensity, followed by protocol exercises, as described (see Additional file 1).

Mandatorily, during the first 2 weeks of treatment and/or in the presence of pain, the proposed exercises 


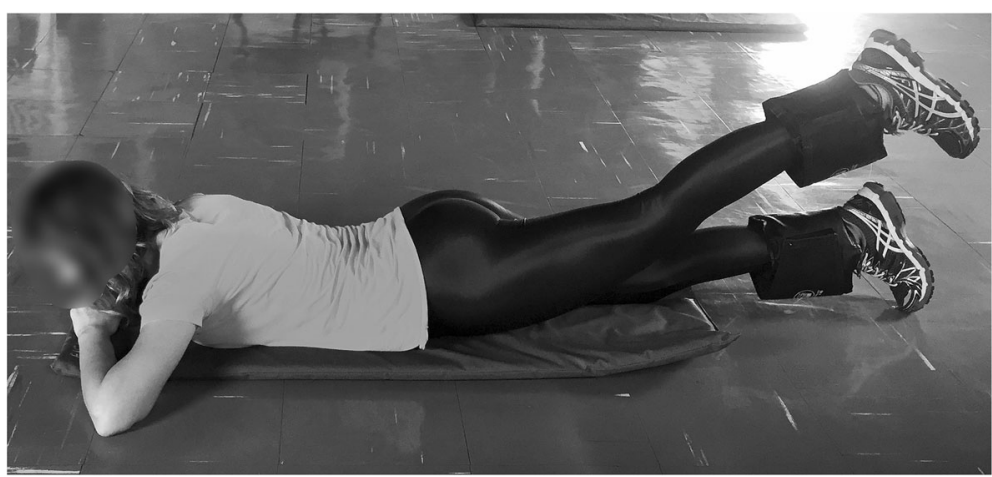

Fig. 5 Hip Extension in prone position (1st-12th week). This exercises will be performed by the Strengthening Group (SG) and Neuromuscular Training Group (NMTG). (Originals photos and own authorship)

will be performed within the "protection range" of the patellofemoral joint, with $90-45^{\circ}$ of knee flexion during exercises in Open Kinetic Chain $(\mathrm{OKC})$ and $0-45^{\circ}$ of knee flexion during Closed Kinetic Chain (CKC) exercises [39]. With the exception of squatting ("wall squat"), it will be performed between $0^{\circ}$ and $60^{\circ}$ of knee flexion [40]. From the third week, the range of motion of knee flexion will be progressed (in exercises "seated knee extension," "leg press," and "wall squat"). This progression will only be performed when the patient has no pain (VAS score 0) or feels only discomfort (VAS score 1) above the protection range of the patellofemoral joint. If the patient experiences pain (VAS score 2 or more) when progressing through the range of motion, the patient will be asked to perform the exercise as much as possible in a pain-free or discomforting (VAS score 0-1) range of motion. If unable to increase the range of motion the exercise will be kept within the protection range

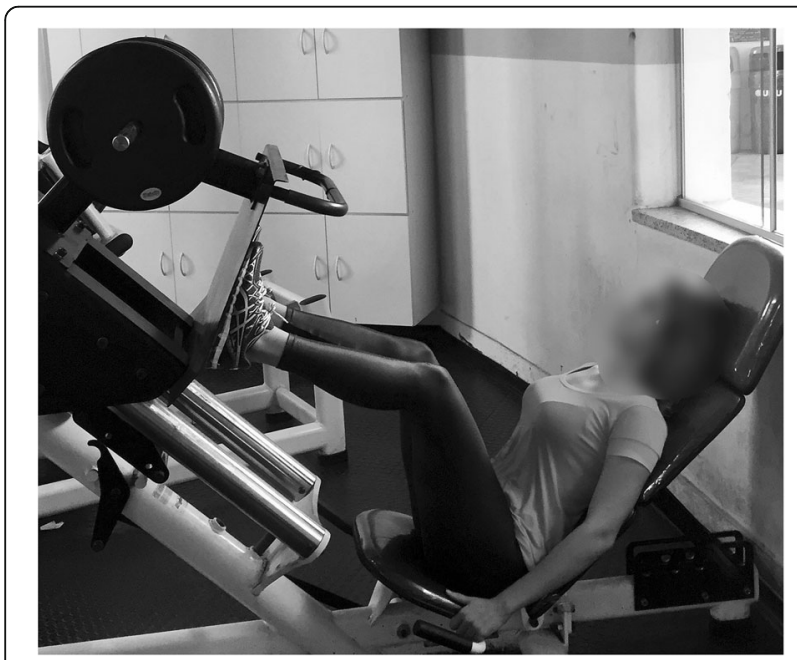

Fig. 6 Leg Press $45^{\circ}$ (1st-12th week). This exercise will be performed by the Strengthening Group (SG) and Neuromuscular Training Group (NMTG). (Originals photos and own authorship) of the patellofemoral joint $\left(90-45^{\circ}\right.$ for $\mathrm{OKC}$; and 0 $45^{\circ}$ for CKC) until the pain has resolved.

The initial load during training will be standardized at $70 \%$ of 1 repetition maximum (1RM) - defined as the maximum load at which a patient can perform only one repetition of the exercise with good quality and without pain or the need to compensate. One repetition maximum of the exercises will be determined on the first day of care and revised each week for possible adjustments and changes in the load for all exercises. Only the "wall squat" exercise will be started with a load corresponding to $10 \%$ of the body weight of each patient.

Exercise loads will be increased weekly starting from the third week. There is a standard progression model, which corresponds to a $5-10 \%$ increase in current load. The criteria used for progression are: (1) the presence of full range of motion with VAS score $0-1$; (2) performing the same exercise without the need to compensate. These criteria were established based on the protocol described by Baldon et al. [33]. CarciBAND ${ }^{\circ}$ elastic bands (São Paulo, Brazil), a balance disc from ACTE Sports $^{\circ}$ (www.actesports.com), model T6-AZ, and a Physicus $^{\circ}$ (Neighborhood Limoeiro) trampoline will be used in this study.

The exercise program for both groups will consist of three sets of 10 repetitions. The exercise on the "board" will be performed with three repetitions in which the patient should maintain the posture for a maximum time that can be achieved. After each set, a rest interval of 12 min will be established. In the presence of moderate to severe pain during exercise (minimum VAS score 5), the session will be interrupted and analgesic measures will be performed immediately.

All patients will be instructed not to perform any other type of treatment for knee pain and to maintain their daily life activities without performing any resisted lower-limb exercise outside the study sessions.

All patients will be informed of inappropriate movement changes in the lower limb during the exercises. In 


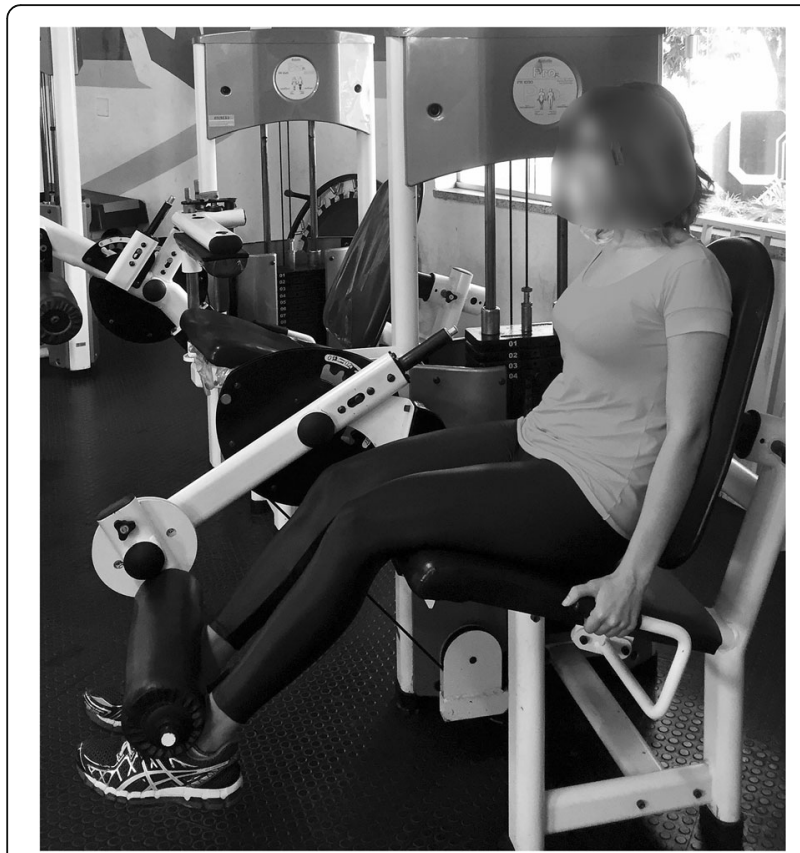

Fig. 7 Seated Knee Extension (1st-12th week). This exercises will be performed by the Strengthening Group (SG) and Neuromuscular Training Group (NMTG). (Originals photos and own authorship) addition, they will be educated to correct any alignment of the lower limb during the exercises through verbal commands and visual feedback.

The minimum rest period between the weekly sessions will be about $48 \mathrm{~h}$.

\section{Outcome measures}

In this study, four outcome measures evaluated before and after the intervention will be used.

\section{Primary outcomes}

The primary outcomes correspond to the pain intensity measured by the VAS in three domains: over the last month, squat $90^{\circ}$, and step of $26 \mathrm{~cm}$ during $1 \mathrm{~min}$ ); functional capacity through two questionnaires: the Anterior Knee Pain Scale (AKPS) and the Activities of Daily Living Scale (ADLS); and two-dimensional (2D) kinematics of the trunk, hip, and lower limb during the single-leg squat.

\section{Secondary outcomes}

The secondary outcomes correspond to the maximum isometric muscle force measured by the manual dynamometer (Lafayette Instrument Company, Lafayette, IN, USA) and the level of satisfaction of the patient in relation to the treatment received, through two multiple choice questions.

Each outcome measure is described below:

\section{Pain assessment}

Pain will be assessed through the VAS [41, 42], with a scale of 0 (without pain) to 10 (extreme pain). The VAS will be applied considering two moments: (1) pain in the last month (current moment); and (2) before and after performing two functional activities: bilateral squatting at $90^{\circ}$ (for $1 \mathrm{~min}$ ) and step-up/down $26 \mathrm{~cm}$ (for $1 \mathrm{~min}$ ).

The VAS is responsive, sensitive and valid to evaluate the PFP population [41, 42].

\section{Functional assessment}

To evaluate function, we will use the ADLS [43-45] and the AKPS [44, 46, 47] questionnaires. These instruments are considered reliable, responsive and valid for the population with PFP $[44,47]$. The ADLS consists of three questions that measure the individual's overall function level on a scale of 0-100. In addition, it has 14 items, which generally measure the symptoms and functional limitations caused by PFP in daily life activities. Moreover, AKPS has 13 items, which also evaluate the symptoms and functional limitations that are often

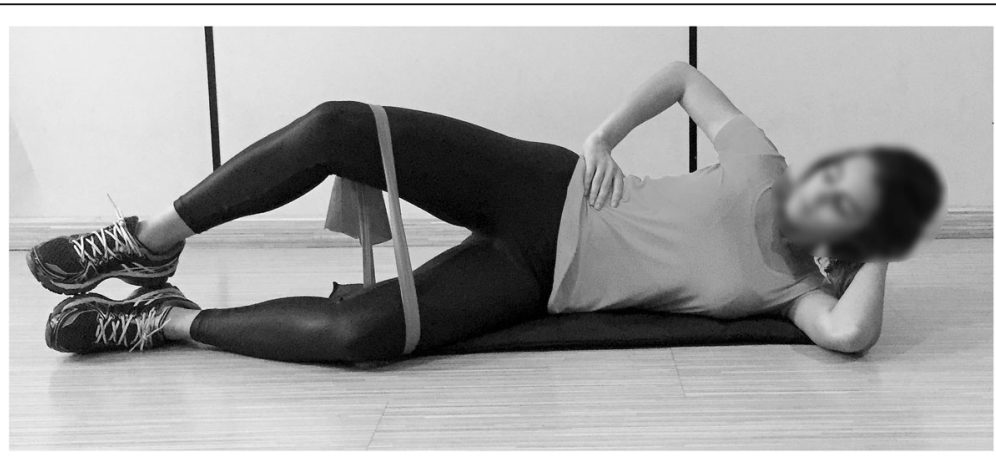

Fig. 8 "Clam" (1stミ6th week). 1st week: elastic band strong average; 2st-3th week: elastic band strong; 4st-5th week: elastic band super strong; From the 6th week: elastic band extra strong. This exercise will be performed by the Strengthening Group (SG) and Neuromuscular Training Group (NMTG). (Originals photos and own authorship) 


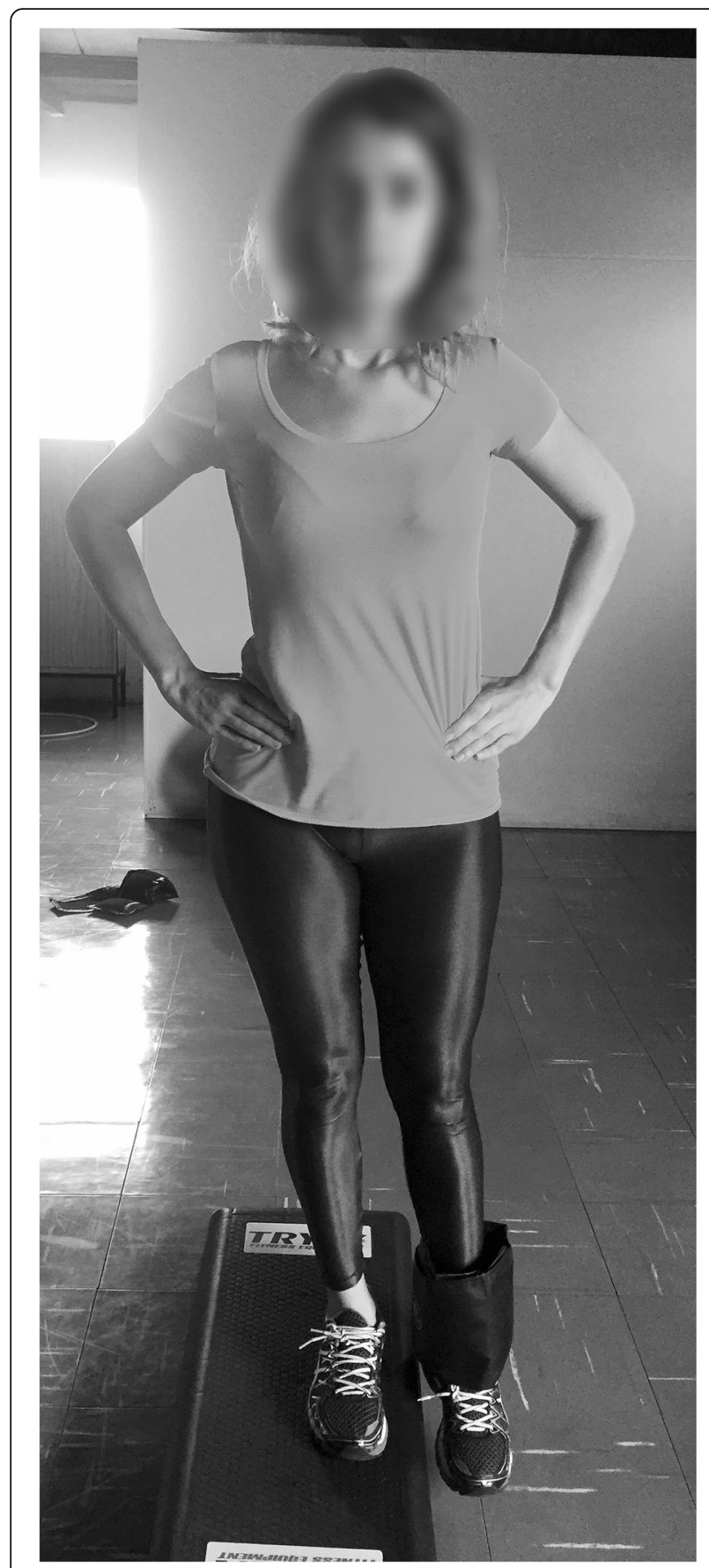

Fig. 9 Pelvic Drop in standing (气6st-12th week). This exercise will be performed by the Strengthening Group (SG) and Neuromuscular Training Group (NMTG). (Originals photos and own authorship)

present in individuals with PFP. In both questionnaires, the score ranges from 0 to 100 , where 0 corresponds to the greatest functional impairment in relation to pain and 100 indicates no functional impairment.

Kinematic assessment

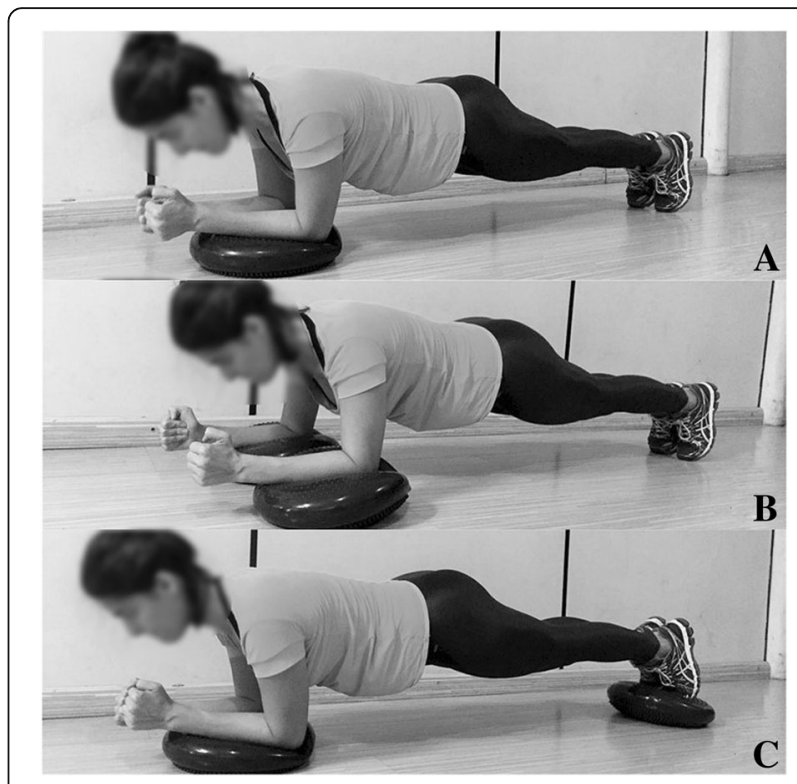

Fig. 10. Ventral plank on an unstable surface (4th-12th week). A) 4st-6th week. B) 7st-9th week. C) 10st-12th week. These exercises will be performed only by the Neuromuscular Training Group (NMTG). (Originals photos and own authorship)

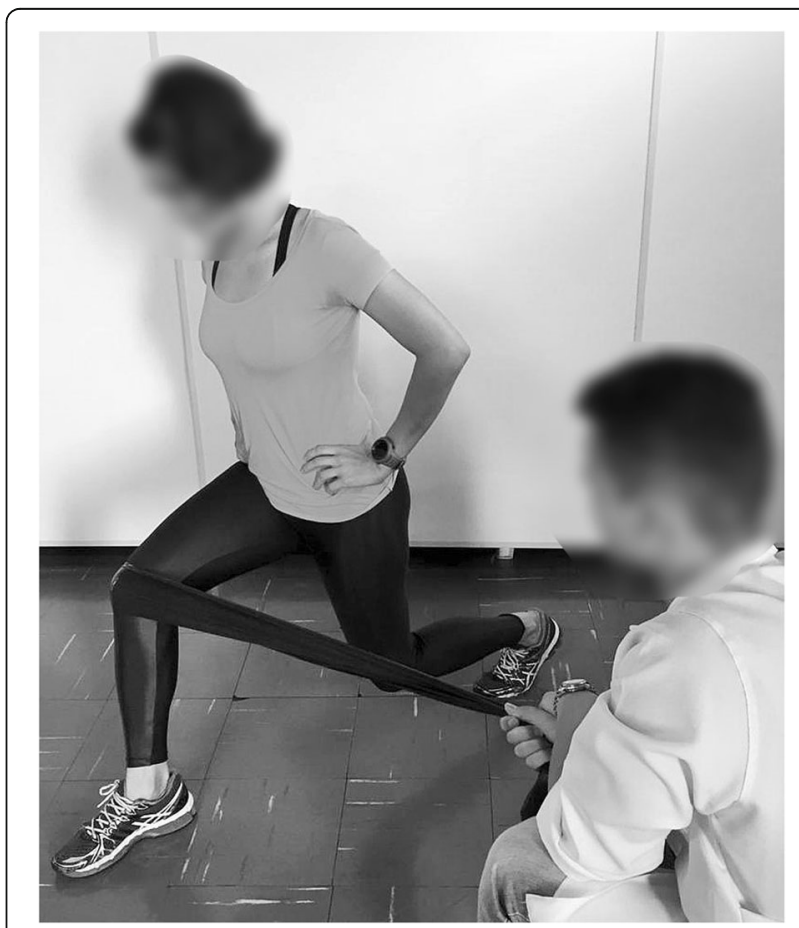

Fig. 11 Lunge with elastic band (4th-12th week). 4st-6th week: elastic band strong average; 7st-9th week: elastic band strong; 10st-12th week: elastic band super strong. This exercise will be performed only by the Neuromuscular Training Group (NMTG). (Originals photos and own authorship) 


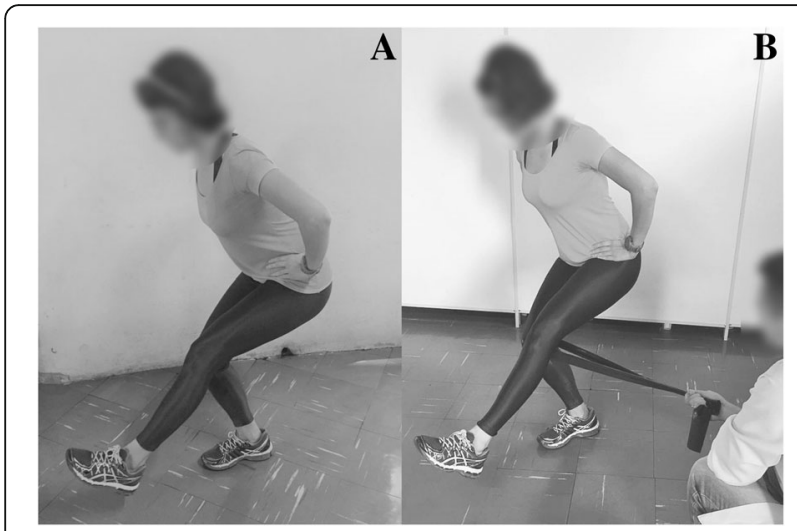

Fig. 12 Single Leg Squat on a stable surface (4th-7th week). A) 4st5th week: without elastic band; B) 6th week: elastic band medium strong, and 7th week: elastic band strong. This exercise will be performed only by the Neuromuscular Training Group (NMTG). (Originals photos and own authorship)

In the frontal and sagittal planes, the lower-limb kinematics will be evaluated during squatting [14, 22, 24, 33] through 2D shooting with two full HD camcorders (JVC GZ-E10, JVC, Wayne, NJ, USA). The cameras will be

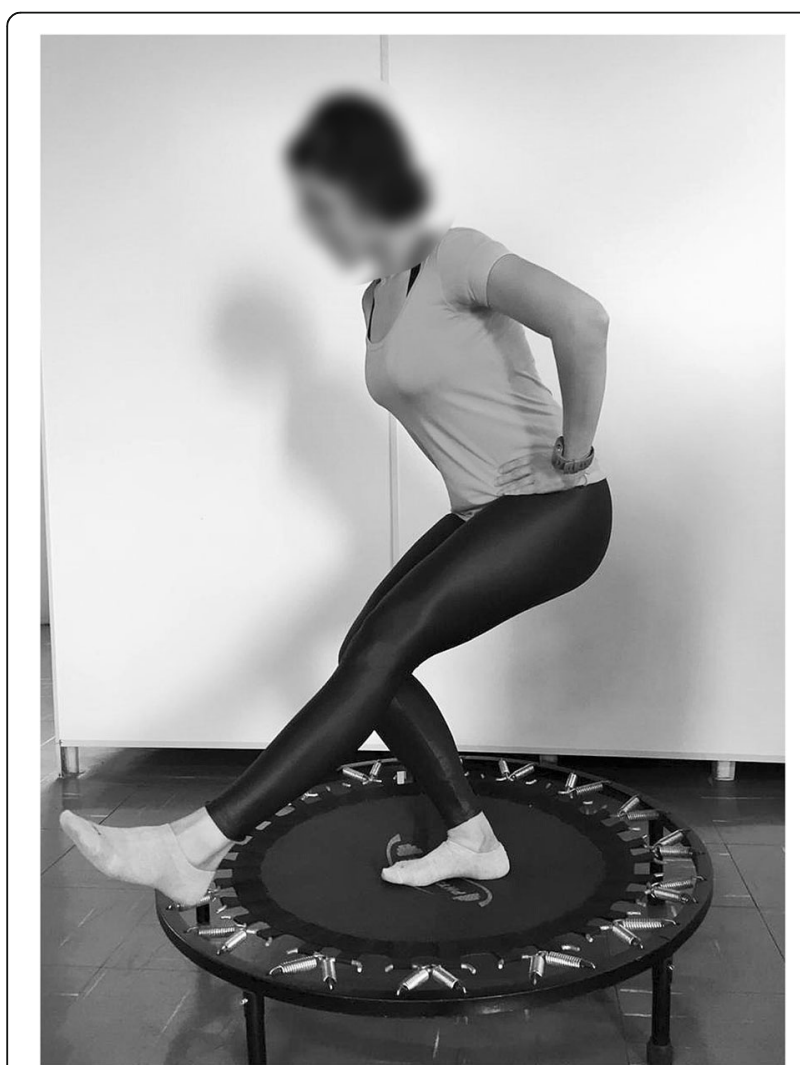

Fig. 13 Single Leg Squat on unstable surface (8th-10th week). This exercise will be performed only by the Neuromuscular Training Group (NMTG). (Originals photos and own authorship)

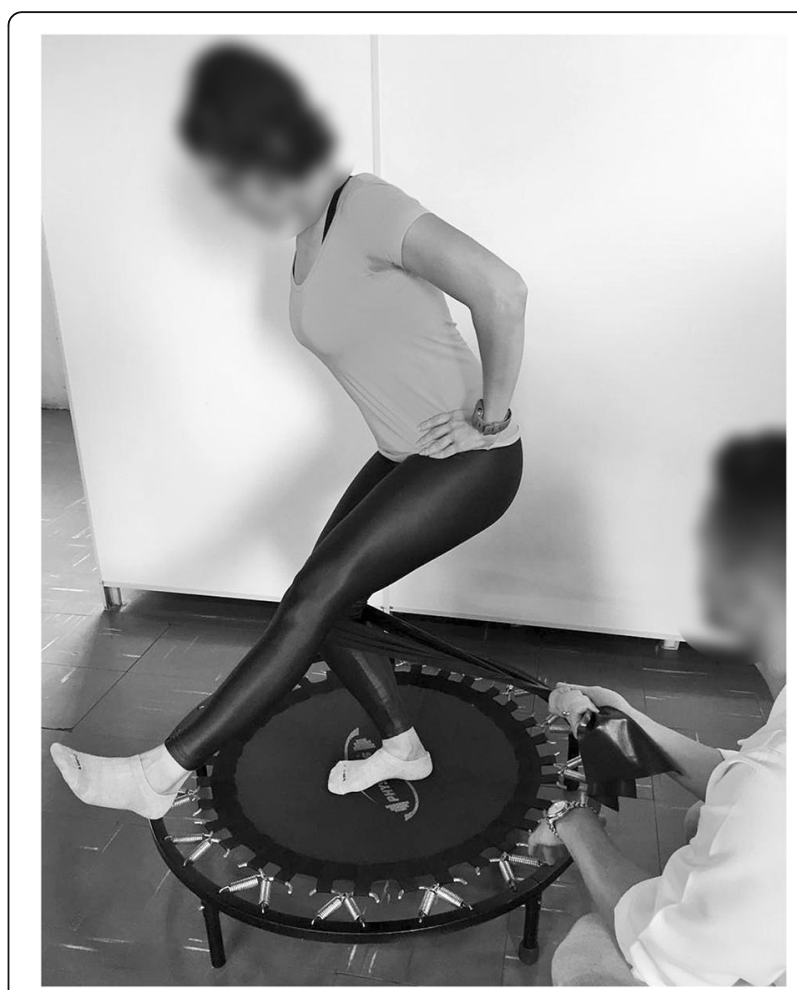

Fig. 14 Single Leg Squat on an unstable surface with elastic band (11th12th week). 11th week: elastic band strong; 12th week: elastic band super strong. This exercise will be performed only by the Neuromuscular Training Group (NMTG). (Originals photos and own authorship)

positioned frontal and lateral to the participant, at a distance of $2.5 \mathrm{~m}$ [48]. Evaluation of both lower limbs will be performed.

The static calibration of the system will be performed using a $160-\mathrm{cm}$-long stick. Subsequently, the patient's bipodal static position will be recorded.

Self-adhesive labels will be positioned bilaterally in the following points: lateral malleolus and medial and anterior tibial tuberosities, lateral and medial epicondyles of the femur and anterior superior iliac spine, upper region of the iliac crest, and spinous process of the vertebra (chest $\mathrm{T} 7$, cervical vertebra $\mathrm{C} 7$, and the centre of the sternum).

To perform the single-leg squat test, the participants will remain in unipodal support with the contralateral limb to the side with the knee flexed at $90^{\circ}$ and arms positioned at the waist. Deep squats will be performed without the contralateral foot touching the ground. The minimum squat angulation is $60^{\circ}$. If, during the test, some of the requirements are not fulfilled, the test will be invalidated and repeated.

For familiarization, the participant will be asked to squat twice with each leg, with a rest interval of $2 \mathrm{~min}$ between each movement. After familiarization, the participants will perform the single-leg squat three times consecutively, in which the average of the measured 


\begin{tabular}{|c|c|c|c|c|c|c|c|c|}
\hline & \multicolumn{8}{|c|}{ STUDY PERIOD } \\
\hline \multirow[b]{2}{*}{ TIMEPOINT $^{\star *}$} & Enrolment & Allocation & \multicolumn{5}{|c|}{ Post-allocation } & \multirow{2}{*}{$\begin{array}{c}\text { Close-out } \\
t_{x}\end{array}$} \\
\hline & $-t_{1}$ & 0 & $t_{1}$ & $t_{2}$ & $t_{3}$ & $t_{4}$ & etc. & \\
\hline \multicolumn{9}{|l|}{ ENROLMENT: } \\
\hline \multirow{2}{*}{ Eligibility screen } & $\mathrm{X}$ & & & & & & & \\
\hline & $\mathrm{X}$ & & & & & & & \\
\hline Randomization & $\mathrm{x}$ & & & & & & & \\
\hline Recruitment & $\mathrm{X}$ & & & & & & & \\
\hline Allocation & & $x$ & & & & & & \\
\hline \multicolumn{9}{|l|}{ INTERVENTIONS: } \\
\hline \multicolumn{9}{|l|}{ [Intervention A - Strengthening Group] } \\
\hline \multicolumn{9}{|l|}{$\begin{array}{r}\text { [Intervention B - Neuromuscular } \\
\text { Training Group] }\end{array}$} \\
\hline \multicolumn{9}{|l|}{$\begin{array}{l}\text { ASSESSMENTS: } \\
\end{array}$} \\
\hline \multicolumn{9}{|l|}{$\begin{array}{r}\text { [Baseline variables: Height, Weight, } \\
\text { age, evaluation of pain, function, } \\
\text { strength of hip and quadriceps, } \\
\text { kinematic of trunk and lower limb] }\end{array}$} \\
\hline $\begin{array}{l}\text { [Outcome variables: evaluation of } \\
\text { pain, function, strength of hip and } \\
\text { quadriceps, kinematic of trunk and } \\
\text { lower limb, level of satisfaction] }\end{array}$ & $x$ & & & & & & & $x$ \\
\hline
\end{tabular}

Fig. 15 Schematic diagram wich depict the overall schedule and time commitment for trial participants

angles is considered for analysis. In the frontal plane, the following angles will be measured: dynamic valgus of the knee, pelvic tilt, and lateral flexion of the trunk. In the axial planes, the angles of anterior trunk flexion and knee flexion will be measured. The analysis of these angles will be performed by the Kinovea ${ }^{\oplus}$ program.

\section{Strength evaluation}

The measurement of the strength of knee extensors [49], abductors [15], lateral rotators [15], and hip extensors [15] will be obtained by means of an evaluation using the manual dynamometer (Lafayette Instrument Company, Lafayette, IN, USA) [50, 51]. An instrument that is valid and reliable [50].

To measure the strength of the knee extensors, the individual will be seated at the edge of the stretcher with the hips flexed at $90^{\circ}$ and the knee to be tested at $60^{\circ}$. For the abductors, the individual will be positioned lying on their side, and the limb to be tested will be facing upwards, with neutral rotation of the hip, $10^{\circ}$ of extension, and $20^{\circ}$ of abduction [15]. For the lateral rotators, the individual will be seated at the edge of the stretcher with hips and knees flexed at $90^{\circ}$ and will be oriented to perform slight lateral rotation so that the lateral malleolus is aligned with the midline of the body [15]. For the hip extensors, the individual will be in the prone position with the knee to be tested having $90^{\circ}$ of flexion and $10^{\circ}$ of extension [15].

The use of a stabilizing strap will be adopted during the tests to avoid the need to compensate, and to stabilize the dynamometer. Before performing the tests, the patient will be asked to perform two submaximal isometric contractions of each muscle group, for familiarization, with a 1-min rest to start the test. The patient will be asked to perform three maximum voluntary isometric contractions of each muscle group for $5 \mathrm{~s}$. A 1-min rest period will be allowed between each measurement. During the conduct of all tests, the patient will be verbally encouraged using the words "Force, force, force!" For the analysis, we will consider the average strength of the test of each muscle group.

\section{Level of satisfaction}

This parameter will be measured during the reassessment of the patient, using a qualitative questionnaire composed of two multiple choice questions, elaborated exclusively by the principal investigator of the study. The purpose of this questionnaire was to measure the patient's satisfaction with the treatment and their current clinical condition [6]. 


\section{Data analysis}

The Kolmogorov-Smirnov normality test will be performed. If the null hypothesis is confirmed, parametric data for the comparisons will be used. However, if the null hypothesis is not confirmed, nonparametric tests will be conducted.

For parametric data, comparison between the groups will be carried out using linear mixed models, considering $P$ values $\leq 0.05$ as significant difference. For intragroup comparison (pre and post intervention), the analysis of variance (ANOVA) test will be used for repeated measurements, considering $P$ values $\leq 0.05$ as significant difference.

The clinical relevance of the results will be confirmed by calculating the effect size (Cohen's d) of the significant differences. The following effects will be considered: 0.00-0.49, small; $0.50-0.79$, medium; and above 0.80 , large (Cohen, 1988). An intention-to-treat analysis will be performed [52].

\section{Discussion}

Although the protocol for strengthening the hip and knee muscles has already been well established in the literature and is considered "gold standard" conservative treatment in individuals with PFP [34] because of its effectiveness in improving pain, function, and the kinematics of the lower limb [34], the effects of neuromuscular training on the pattern of lower-limb movement and the addition of these exercises in the physiotherapeutic treatment program remains less well discussed and understood [37].

In view of this, the results of the present study may contribute to the decision-making for the physiotherapeutic intervention of patients with PFP by providing information on the effects of neuromuscular training on the clinical and kinematic conditions in this population Additional file 2.

\section{Additional files}

Additional file 1: This additional file describes in detail the exercises of the proposed protocol for both study groups. (DOCX $21 \mathrm{~kb}$ )

Additional file 2: Standard Protocol Items: Recommendations for Interventional Trials (SPIRIT) 2013 Checklist: recommended items to address in a clinical trial protocol and related documents*. (PDF $119 \mathrm{~kb}$ )

\section{Abbreviations}

ADLS: Activities of Daily Living Scale; AKPS: Anterior Knee Pain Scale; CAPES: Coordenação de Aperfeiçoamento de Pessoal de Nível Superior: NMTG: Neuromuscular Training Group; PFP: Patellofemoral pain; SG: Strengthening Group; UFU: Universidade Federal de Uberlândia; VAS: Visual Analog Scale

\section{Acknowledgements}

The authors would like to thank the Universidade Federal de Uberlândia (UFU) for providing the assessment and treatment facilities used in the present study. In addition, we would also like to thank CAPES for funding the development of this research.
Trial status

Protocol version number and date: version 03/date 20 February 2019. Patient recruitment is ongoing;

Date recruitment began: 28 January 2018;

Approximate date when recruitment will be completed: 31 July 2019.

\section{Authors' contributions}

NCS and LRF developed the concept and design of the protocol. LRF performed the randomization and random allocation of the volunteers to the groups. NCS, MGG, MCS, and MBON participated in the execution of the project protocol, with MCS as the blinded evaluator and others performing the interventions. NCS and LRF will analyze and interpret the data. NCS drafted the manuscript with LRF's critical contributions. All authors have read and approved the final manuscript.

\section{Funding}

This study was funded by the Coordenação de Aperfeiçoamento de Pessoal de Nivel Superior (CAPES), Brazil - Finance Code 1799120. This funding source had no role in the design of this study and will not have any role during its execution, analyses, interpretation of the data, or the decision to submit the results.

\section{Availability of data and materials}

Data sets generated and/or analyzed during the current study may be made available by the corresponding author upon reasonable request.

\section{Ethics approval and consent to participate}

This study was approved by the Research Ethics Committee of the Universidade Federal de Uberlândia (CEP-UFU), under protocol number CAAE: 57621316.0.0000.5152. The Consent Form (written) to participate this study will be obtained from all participants.

\section{Consent for publication}

All participants will receive a Consent Form to be included in the study. By signing this form, the participants allow the use and publication of their data anonymously in this study. The (written) Consent Form allowing the use and publication of their images (Figs. 3, 4, 5, 6, 7, 8, 9, 10, 11, 12, 13, 14 and 15) was obtained from these individuals.

\section{Competing interests}

The authors declare that they have no competing interests.

Received: 20 February 2019 Accepted: 12 August 2019

Published online: 11 October 2019

\section{References}

1. Smith BE, Selfe J, Thacker D, Hendrick P, Bateman M, Moffatt F, Rathleff MS, Smith TO, Logan P. Incidence and prevalence of patellofemoral pain: a systematic review and meta-analysis. PloS one. 2018;13(1):e0190892.

2. Thomeé R, Augustsson J, Karlsson J. Patellofemoral pain syndrome: a review of current issues. Sports Med. 1999;28(4):245-62.

3. Witvrouw E, Callaghan MJ, Stefanik J, Noehren B, Bazett-Jones D, Willson JD, Earl-Boehm J, Davis IS, Powers CM, McConnel J, Crossley KM. Patellofemoral pain: consensus treatment from the 3rd International Patellofemoral Pain Research Retreat held in Vancouver, September 2013. Br J Sports Med. 2014; 48(6):411-4.

4. Utting MR, Davies $G$, Newman JH. Is anterior knee pain a predisposingfactor to patellofemoral osteoarthritis? Knee. 2005;12(5):362-5.

5. Crossley KM. Is patellofemoral osteoarthritis a common sequela of patellofemoral pain? Br J Sports Med. 2014:48:409-10.

6. Crossley KM, Van Middelkoop M, Callaghan MJ, Collins NJ, Rathleff MS, Barton CJ. 2016 Patellofemoral Pain Consensus Statement from the 4th International Patellofemoral Pain Research Retreat, Manchester. Part 2: recommended physical interventions (exercise, taping, bracing, foot orthoses and combined interventions). Br J Sports Med. 2016;48(6):844-52.

7. Neal BS, Lack SD, Lankhorst NE, Raye A, Morrissey D, Middelkoop MV. Risk factors for patellofemoral pain: a systematic review and meta-analysis. $\mathrm{Br} \mathrm{J}$ Sports Med. 2019:53(5):270-81.

8. Powers CM, Witvrouw E, Davis IS, Crossley KM. Evidence-based framework for a pathomechanical model of patellofemoral pain: 2017 patellofemoral pain consensus statement from the 4th International Patellofemoral Pain Research Retreat, Manchester, UK: part 3. Br J Sports Med. 2017;51(24):1713-23. 
9. Vicenzino B, Maclachlan L, Rathleff MS. Taking the pain out of the patellofemoral joint: articulating a bone of contention. Br J Sports Med. 2019;53(5):268-269.

10. Maclachlan LR, Collins NJ, Matthews MLG, Hodges PW, Vicenzino B. The psychological features of patellofemoral pain: a systematic review. $\mathrm{Br} J$ Sports Med. 2017:51(9):732-42.

11. Crossley KM, Middelkoop MV, Barton CJ, Culvenor A. rethinking patellofemoral pain: prevention, management and long-term consequences. Best Pract Res Clin. Rheumatol. 2019;33(1):48-65.

12. Cowan SM, Crossley KM, Bennell KL. Altered hip and trunk muscle function in individuals with patellofemoral pain. Br J Sports Med. 2009;43:584-8.

13. Willson JD, Davis IS. Lower extremity strength and mechanics during jumping in women with patellofemoral pain. J Sport Rehabil. 2009;18(1):76-90.

14. Nakagawa TH, Moriya ET, Maciel CD, Serrão FV. Trunk, pelvis, hip, and knee kinematics, hip strength, and gluteal muscle activation during a single-leg squat in males and females with and without patellofemoral pain syndrome. J Orthop Sports Phys Ther. 2012;42(6):491-501.

15. Magalhaes E, Fukuda TY, Sacramento SN, Forgas A, Cohen M, Abdalla RJ. A comparison of hip strength between sedentary females with and without patellofemoral pain syndrome. J Orthop Sports Phys Ther. 2010;40(10):641-7.

16. Prins MR, Van Der Wurff P. Females with patellofemoral pain syndrome have weak hip muscles: a systematic review. Aust J Physiother. 2009;55(1):9-15.

17. Magalhães E, Silva APMCC, Sacramento SN, Martin RL, Fukuda TY. Isometric strength ratios of the hip musculature in females with patellofemoral pain: a comparison to pain free controls. J Strength Cond Res. 2013;27(8):2165-70.

18. Pappas E, Wong-Tom WM. Prospective predictors of patellofemoral pain syndrome: a systematic review with meta-analysis. Sports Health. 2012;4(2): $115-20$.

19. Dierks TA, Manal KT, Hamill J, Davis IS. Proximal and distal influences on hip and knee kinematics in runners with patellofemoral pain during a prolonged run. J Orthop Sports Phys Ther. 2008;38(8):448-56.

20. Dos Reis AC, Correa JCF, Bley AS, Rabelo NDDA, Fukuda TY, Lucareli PRG. Kinematic and kinetic analysis of the single-leg triple hop test in women with and without patellofemoral pain. J Orthop Sports Phys Ther. 2015; 45(10):799-807.

21. Schwane BG, Goerger BM, Goto S, Blackburn JT, Aguilar AJ, Padua DA. Trunk and lower extremity kinematics during stair descent in women with or without patellofemoral pain. J Athl Train. 2015;50(7):704-12

22. Bley AS, Correa JCF, Dos Reis AC, Rabelo NDDA, Marchetti PH, Lucareli PRG. Propulsion phase of the single leg triple hop test in women with patellofemoral pain syndrome: a biomechanical study. PLoS One. 2014;9(5):e97606.

23. Herrington L. Knee valgus angle during single leg squat and landing in patellofemoral pain patients and controls. Knee. 2014;21(2):514-7.

24. Salsich GB, Graci V, Maxam DE. The effects of movement pattern modification on lower extremity kinematics and pain in women with patellofemoral pain. J Orthop Sports Phys Ther. 2012;42(12):1017-24.

25. Fulkerson JP, Hungerford DS. Patellar tilt/compression and the excessive lateral pressure syndrome (ELPS). Disorders of the patellofemoral joint. 2nd ed. Baltimore: Williams and Wilkins; 1990. p. 102-23.

26. Nakagawa TH, Serrão FV, Maciel CD, Powers CM. Hip and knee kinematics are associated with pain and self-reported functional status in males and females with patellofemoral pain. Int J Sports Med. 2013;34(11):997-1002.

27. Holden S, Boreham C, Doherty C, Delahunt E. Two-dimensional knee valgus displacement as a predictor of patellofemoral pain in adolescent females. Scand J Med Sci Sports. 2015;27(2):88-194.

28. Nakagawa TH, Muniz TB, Baldon RDM, Macial CD, Reiff RBM, Serrão FV. The effect of additional strengthening of hip abductor and lateral rotator muscles in patellofemoral pain syndrome: a randomized controlled pilot study. Clin Rehabil. 2008;22(12):1051-60.

29. Fukuda TY, Rossetto FM, Magalhães E, Bryk FF, Lucareli PRG, Carvalho NAA. Short-term effects of hip abductors and lateral rotators strengthening in females with patellofemoral pain syndrome: a randomized controlled clinical trial. J Orthop Sports Phys Ther. 2010; 40(11):736-42.

30. Earl JE, Hoch AZ. A proximal strengthening program improves pain, function, and biomechanics in women with patellofemoral pain syndrome. Am J Sports Med. 2011;39(1):154-63.

31. Fukuda TY, Melo WP, Zaffalon BM, Rossetto FM, Magalhães E, Bryk FF, Martin RL. Hip posterolateral musculature strengthening in sedentary women with patellofemoral pain syndrome: a randomized controlled clinical trial with 1year follow-up. J Orthop Sports Phys Ther. 2012;42(10):823-30.
32. Mascal CL, Landel L, Powers CM. Management of patellofemoral pain targeting hip, pelvis, and trunk muscle function: 2 case reports. J Orthop Sports Phys Ther. 2003;33(11):647-60.

33. Baldon RDM, Serrão FV, Silva RS, Piva SR. Effects of functional stabilization training on pain, function, and lower extremity biomechanics in women with patellofemoral pain: a randomized clinical trial. J Orthop Sports Phys Ther. 2014;44(4):240-A8.

34. Nascimento LR, Teixeira-Salmela LF, Souza RB, Resende RA. Hip and knee strengthening is more effective than knee strengthening alone for reducing pain and improving activity in individuals with patellofemoral pain: a systematic review with meta-analysis. J Orthop Sports Phys Ther. 2018;48(1):19-31.

35. Noehren B, Scholz J, Davis I. The effect of real-time gait retraining on hip kinematics, pain and function in subjects with patellofemoral pain syndrome. Br J Sports Med. 2011;45:691-6.

36. Willyv RW, Scholz JP, Davis IS. Mirror gait retraining for the treatment of patellofemoral pain in female runners. Clin Biomech. 2012;27:1045-51.

37. Dos Anjos Rabelo ND, Costa LOP, De Lima BM, Dos Reis AC, Bley AS, Fukuda TY, Lucareli PRG. Adding motor control training to muscle strengthening did not substantially improve the effects on clinical or kinematic outcomes in women with patellofemoral pain: a randomised controlled trial. Gait Posture. 2017;58:280-6.

38. da Silva GDSF, Bergamaschine R, Rosa M, Melo C, Miranda R, Filho MB. Avaliação do nível de atividade física de estudantes de graduação das áreas saúde/biológica. Rev Bras Med Esporte. 2007;13(1):39-42.

39. Steinkamp LA, Dillingham MF, Markel MD, Hill JA, Kaufman KR. Biomechanical considerations in patellofemoral joint rehabilitation. Am J Sports Med. 1993;21(3):438-44.

40. Bevilaqua-Grossi D, Felicio LR, Simões R, Coqueiro KRR, Monteiro-Pedro V. Avaliação eletromiográfica dos músculos estabilizadores da patela durante exercício isométrico de agachamento em indivíduos com síndrome da dor femoropatelar. Rev Bras Med Esporte. 2005;11(3):159-63.

41. Crossley KM, Bennell KL, Cowan SM, Green S. Analysis of outcome measures for persons with patellofemoral pain: which are reliable and valid? Arch Phys Med Rehabil. 2004;85(5):815-22.

42. Chesworth BM, Culham EG, Tata GE, Peat M. Validation of outcome measures in patients with patellofemoral syndrome 1. J Orthop Sports Phys Ther. 1989;10(8):302-8.

43. Irrang JJ, Snyder-Mackler L, Wainner RS, Fu FH, Harner CD. Development of a patient-reported measure of function of the knee. J Bone Joint Surg Am. 1998;80(8):1132-45.

44. Esculier JF, Roy JS, Bouyer LJ. Psychometric evidence of self-reported questionnaires for patellofemoral pain syndrome: a systematic review. Disabil Rehabil. 2013;35(26):2181-90.

45. Piva SR, Gil AB, Moore CG, Fitzgerald GK. Responsiveness of the activities of daily living scale of the knee outcome survey and numeric pain rating scale in patients with patellofemoral pain. J Rehabil Med. 2009;41(3):129-35.

46. Kujala UM, Jaakkola LH, Koskinen SK, Taimela S, Hurme M, Nelimarkka O. Scoring of patellofemoral disorders. Arthroscopy. 1993:9(2):159-63.

47. Da Cunha RA, Costa LOP, Hespanhol LC, Pires RS, Kujala UM, Lopes AD. Translation, cross-cultural adaptation, and clinimetric testing of instruments used to assess patients with patellofemoral pain syndrome in the Brazilian population. J Orthop Sports Phys Ther. 2013;43(5):332-9.

48. lunes DH, Castro FA, Salgado HS, Moura IC, Oliveira AS, Bevilaqua-Grossi D. Confiabilidade intra e interexaminadores e repetibilidade da avaliação postural pela fotogrametria. Rev Bras Fisioter. 2005;9(3):327-34.

49. Bolgla LA, Malone TR, Umberger BR, Uhl TL. Comparison of hip and knee strength and neuromuscular activity in subjects with and without patellofemoral pain syndrome. Int J Sports Phys Ther. 2011;6(4):285.

50. Hébert LJ, Maltais DB, Lepage C, Saulnier J, Crête M, Perron M. Isometric muscle strength in youth assessed by hand-held dynamometry: a feasibility, reliability, and validity study: a feasibility, reliability, and validity study. Pediatr Phys Ther. 2011;23(3):289-99.

51. Robinson RL, Nee RJ. Analysis of hip strength in females seeking physical therapy treatment for unilateral patellofemoral pain syndrome. J Orthop Sports Phys Ther. 2007;37(5):232-8.

52. Elkins MR, Moseley AM. Intention-to-treat analysis. J Physiother. 2015;61(3): $165-7$.

\section{Publisher's Note}

Springer Nature remains neutral with regard to jurisdictional claims in published maps and institutional affiliations. 